epl draft

This is a pre-print of an article published in

Europhysics Letters 129 (2020) 3006

The final authenticated version is available online at:

https://iopscience.iop.org/article/10.1209/0295-5075/129/30006

\title{
Thomas precession and Thomas-Wigner rotation: correct solutions and their implications
}

\author{
AleXANDER KhOLMETSKII ${ }^{1(\mathrm{a})}$, OLEG MisSEVITCH ${ }^{2}$, TOLGA YARMAN $^{3}$, Metin ARIK $^{4}$ \\ ${ }^{1}$ Department of Physics, Belarusian State University - Nezavisimosti Avenue 4, 220030, Minsk, Belarus \\ ${ }^{2}$ Research Institute for Nuclear Problems, Belarusian State University-Bobrujskaya str., 11, 220030, Minsk, Belarus \\ ${ }^{3}$ Okan University, Akfirat, Istanbul, Turkey \\ ${ }^{4}$ Bogazici University, Istanbul, Turkey
}

received and accepted dates provided by the publisher other relevant dates provided by the publisher

PACS 03.30.+p - Special relativity

\begin{abstract}
We address to the Thomas precession for the hydrogenlike atom and point out that in the derivation of this effect in the semi-classical approach, two different successions of rotation-free Lorentz transformations between the laboratory frame $\mathrm{K}$ and the proper electron's frames, $\mathrm{K}_{\mathrm{e}}(t)$ and $\mathrm{K}_{\mathrm{e}}(t+d t)$, separated by the time interval $d t$, were used by different authors. We further show that the succession of Lorentz transformations $\mathrm{K} \rightarrow \mathrm{K}_{\mathrm{e}}(t) \rightarrow \mathrm{K}_{\mathrm{e}}(t+d t)$ leads to relativistically non-adequate results in the frame $\mathrm{K}_{\mathrm{e}}(t)$ with respect to the rotational frequency of the electron spin, and thus an alternative succession of transformations $\mathrm{K} \rightarrow \mathrm{K}_{\mathrm{e}}(t), \mathrm{K} \rightarrow \mathrm{K}_{\mathrm{e}}(t+d t)$ must be applied. From the physical viewpoint this means the validity of the introduced "tracking rule", when the rotation-free Lorentz transformation, being realized between the frame of observation $\mathrm{K}$ and the frame $\mathrm{K}(t)$ co-moving with a tracking object at the time moment $t$, remains in force at any future time moments, too. We apply this rule to the moving macroscopic objects and analyze its implications with respect to the Thomas-Wigner rotation and its application to astrometry.
\end{abstract}

1. Introduction. - The Thomas precession of the electron spin had been introduced in ref. [1] in the semi-classical analysis of spin-orbit coupling in hydrogenlike atoms, where the usual representation about a Larmor precession of the magnetic dipole moment of electron in the presence of magnetic field, existing in the co-moving frames of an orbiting electron, yielded the result being twice larger than the measurement data. This apparent contradiction had been eliminated by Thomas, who pointed out that the successive Lorentz transformations (LT) from the rest frame of nucleus K to the frame $\mathrm{K}_{\mathrm{e}}(t)$ co-moving with the electron at the moment $t$, and then from $\mathrm{K}_{\mathrm{e}}(t)$ to $\mathrm{K}_{\mathrm{e}}(t+d t)$, entail a spatial rotation of coordinate axes of the system $\mathrm{K}_{\mathrm{e}}(t+d t)$ with respect to the system $\mathrm{K}$, later called the Thomas-Wigner rotation [2,3]. The time derivative of the angle of Thomas-Wigner rotation yields the frequency equal to the half of the frequency of Larmor precession $\omega_{L}$ of electron's spin. Hence, the measured spin- orbit interaction in hydrogenlike atom should be half of the value resulting from the frequency of Larmor precession, which agreed with the experimental results.

This disclosure by Thomas has played the important role in favor of the hypothesis about spin [4] and nowadays, the Thomas-Wigner rotation and Thomas precession represent the classical relativistic effects considered in numerous books and papers (see, e.g. refs. [5-13], published already in $21^{\text {st }}$ century; our own contribution is presented in refs. [14-20]).

The Thomas-Wigner rotation and Thomas precession, being purely kinematical effects $[21,22]$, result from noncommutativity of successive rotation-free LT (Lorentz boosts L) between three Lorentz frames $\mathrm{K}, \mathrm{K}_{1}$ and $\mathrm{K}_{2}$, moving with non-collinear relative velocities $\boldsymbol{v}$ (between $\mathrm{K}, \mathrm{K}_{1}$ ) and $\boldsymbol{u}$ (between $\mathrm{K}_{1}, \mathrm{~K}_{2}$ ). Namely, the succession $\mathbf{L}(\boldsymbol{v}) \mathbf{L}(\boldsymbol{u})$, corresponding to the order of transformations $\mathrm{K} \rightarrow \mathrm{K}_{1} \rightarrow \mathrm{K}_{2}$, differs from the direct rotation-free transformation $\mathbf{L}(\boldsymbol{v} \oplus \boldsymbol{u})$

\footnotetext{
(a)E-mail: a1kho1metskii@gmai1 .com
} 
from $\mathrm{K}$ to $\mathrm{K}_{2}$ by the Thomas-Wigner rotation of the system $\mathrm{K}_{2}$ with respect to $K$ at the angle $\theta_{02}$. Alternatively, one can consider another succession of rotation-free transformations $\mathrm{K} \rightarrow \mathrm{K}_{2} \rightarrow \mathrm{K}_{1} \quad\left(\mathbf{L}(\boldsymbol{v} \oplus \boldsymbol{u}) \mathbf{L}^{-1}(\boldsymbol{u})\right)$, which differs from the direct transformation $\mathrm{K} \rightarrow \mathrm{K}_{1}$ by the Thomas-Wigner rotation of the system $K_{1}$ with respect to $K$ at the angle $\theta_{01}$.

Considering a circular motion of the classical electron $e$ around the nucleus $Z e$, resting in the labframe $\mathrm{K}$, we associate the frame $\mathrm{K}_{1}$ with $\mathrm{K}_{\mathrm{e}}(t)$ and the frame $\mathrm{K}_{2}$ with $\mathrm{K}_{\mathrm{e}}(t+d t)$. Designating though $v$ the velocity of $\mathrm{K}_{\mathrm{e}}(t)$ in $\mathrm{K}$, and $\dot{v} d t$ the velocity of $\mathrm{K}_{\mathrm{e}}(t+d t)$ in $\mathrm{K}_{\mathrm{e}}(t)$, we may follow the original approach by Thomas $[1,2]$ and use the successive rotationfree $\mathrm{LT} \mathbf{L}(\boldsymbol{v}) \mathbf{L}(\dot{\boldsymbol{v}} d t)\left(\mathrm{K} \rightarrow \mathrm{K}_{\mathrm{e}}(t) \rightarrow \mathrm{K}_{\mathrm{e}}(t+d t)\right)$.

However, in some other publications (e.g., in the familiar textbook [22]), the Thomas precession is analyzed in another situation, where the rotation-free LT are implemented between the pairs of frames $\mathrm{K}, \mathrm{K}_{\mathrm{e}}(t)$ and $\mathrm{K}, \mathrm{K}_{\mathrm{e}}(t+d t)$, where $\mathrm{K}_{\mathrm{e}}(t)$ and $\mathrm{K}_{\mathrm{e}}(t+d t)$ are no longer related via rotation-free LT, unlike to the adoption by Thomas.

This finding motivated us to analyze closer the available publications about the Thomas precession, where all of them can be indeed divided into two groups:

- the first group, where, following Thomas, the succession of rotation-free $\mathrm{LT} \mathrm{K} \rightarrow \mathrm{K}_{\mathrm{e}}(t) \rightarrow \mathrm{K}_{\mathrm{e}}(t+d t)$ is adopted, which corresponds to the Thomas-Wigner rotation between the systems $\mathrm{K}$ and $\mathrm{K}_{\mathrm{e}}(t+d t)$ (e.g., [1, 2, 5, 21, 23-26];

- the second group, where another succession of rotationfree LT is adopted, $\mathrm{K} \rightarrow \mathrm{K}_{\mathrm{e}}(t), \mathrm{K} \rightarrow \mathrm{K}_{\mathrm{e}}(t+d t)$, which corresponds to the Thomas-Wigner rotation between the systems $\mathrm{K}_{\mathrm{e}}(t)$ and $\mathrm{K}_{\mathrm{e}}(t+d t)$ (e.g., [22, 27-29]).

We notice that none of the publications in both groups contain any argumentation with respect to their choice of succession of LT in the derivation of Thomas precession. Perhaps, this situation can be explained by the fact that for the hydrogenlike atom, considered in the semi-classical limit, both approaches yield identical results with respect to spinorbit coupling in the laboratory frame $\mathrm{K}$.

Nevertheless, in section 2, we clarify the principal difference between both successions of LT mentioned above, and show that relativistically adequate solution in the electron's co-moving frames is derived only under the choice of rotation-free $\mathrm{LT} \mathrm{K} \rightarrow \mathrm{K}_{\mathrm{e}}(t), \mathrm{K} \rightarrow \mathrm{K}_{\mathrm{e}}(t+d t)$.

Extending this result, named below as the "tracking rule", to the motion of macroscopic objects, in section 3, we analyze the problem of the measurement of Thomas-Wigner rotation in Earth laboratory and suggest an appropriate experiment that can serve also as a new method for determination of motional characteristics of Earth and Sun. We conclude in section 4.

\section{Incorrect and correct ways to the Thomas}

precession. - As is known, the mathematical apparatus of relativistic kinematics does not contain any means, which could establish an order of successive rotation-free LT between three inertial reference frames $K_{1}, K_{2}$ and $K_{3}$, moving with respect to each other with non-collinear relative velocities. Usually, this circumstance does not bother researchers, because in the majority of situations, a succession of LT between the frames under consideration is set exogenously, as was done, for example, in all publications on the Thomas precession, starting with [1].

In the case of circular motion of classical electron around immovable nucleus, there are two options for the choice of successive LT under derivation of the Thomas precession, and both of them: $\mathrm{K} \rightarrow \mathrm{K}_{\mathrm{e}}(t) \rightarrow \mathrm{K}_{\mathrm{e}}(t+d t)$ and $\mathrm{K} \rightarrow \mathrm{K}_{\mathrm{e}}(t), \mathrm{K} \rightarrow \mathrm{K}_{\mathrm{e}}(t+d t)$, had been used by researchers, as mentioned above. With respect to a laboratory observer, both options yield the same value of the frequency of Thomas precession. Nevertheless, their physical implications occur different in the electron's comoving frames.

In order to clarify this difference, we address to Fig. 1, where, for simplicity, we show the co-moving electron's frame $\mathrm{K}_{\mathrm{e}}(t)$ at the time moment $t$, when its tangential velocity is parallel to the axis $x$. We also show the co-moving electron's frame $\mathrm{K}_{\mathrm{e}}(t+d t)$ at the time moment $t+d t$, and indicate a spatial turn of the system $\mathrm{K}_{\mathrm{e}}(t+d t)$ around the axis $z$ at the angle $d \theta$.

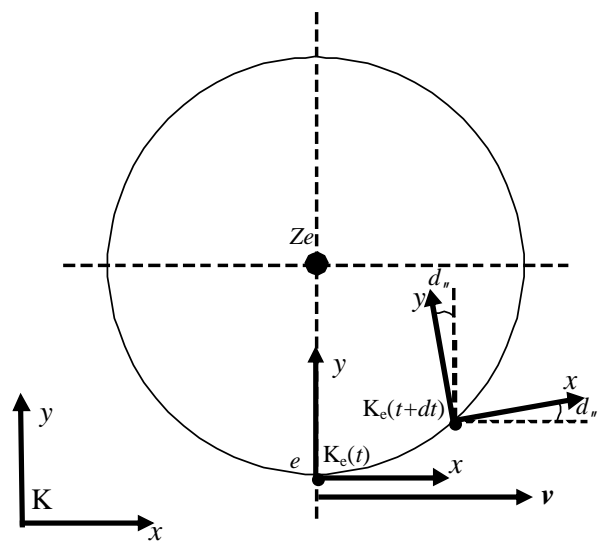

Fig. 1: The rest frame of the nucleus $\mathrm{K}$ (laboratory frame) and the electron co-moving frames $\mathrm{K}_{\mathrm{e}}(t)$ and $\mathrm{K}_{\mathrm{e}}(t+d t)$ at the time moments $t$ and $t+d t$, correspondingly. We show the rotation of the system $\mathrm{K}_{\mathrm{e}}(t+d t)$ at the angle $d \theta$ during the time interval $d t$. At the choice of successive LT $\mathrm{K} \rightarrow \mathrm{K}_{\mathrm{e}}(t) \rightarrow \mathrm{K}_{\mathrm{e}}(t+d t)$, this rotation happens with respect to the laboratory system $\mathrm{K}$; at the choice of succession of $\mathrm{LT}$ $\mathrm{K} \rightarrow \mathrm{K}_{\mathrm{e}}(t), \mathrm{K} \rightarrow \mathrm{K}_{\mathrm{e}}(t+d t)$, this rotation takes place with respect to the system $\mathrm{K}_{\mathrm{e}}(t)$.

Here, one should notice that under the choice of succession of rotation-free LT $\mathrm{K} \rightarrow \mathrm{K}_{\mathrm{e}}(t) \rightarrow \mathrm{K}_{\mathrm{e}}(t+d t)$, the axes of $\mathrm{K}_{\mathrm{e}}(t+d t)$ are turned out with respect to the axes of the labframe $\mathrm{K}$, and remain parallel to the axes of $\mathrm{K}_{\mathrm{e}}(t)$.

When the rotation-free LT are carried out between the frames $\mathrm{K}, \mathrm{K}_{\mathrm{e}}(t)$ and $\mathrm{K}, \mathrm{K}_{\mathrm{e}}(t+d t)$, then the rotation of the system $\mathrm{K}_{\mathrm{e}}(t+d t)$ happens with respect to $\mathrm{K}_{\mathrm{e}}(t)$. In this situation, one may conjecture that in electron's co-moving frames the precession frequency of its spin could be different for two different successions of LT.

Let us show that this is actually the case. 
Namely, under the choice of rotation-free transformations $\mathrm{K} \rightarrow \mathrm{K}_{\mathrm{e}}(t) \rightarrow \mathrm{K}_{\mathrm{e}}(t+d t)$ applied by Thomas [1], an observer in the electron's frame $\mathrm{K}_{\mathrm{e}}(t)$ does not observe any rotation of the frame $\mathrm{K}_{\mathrm{e}}(t+d t)$, so that the corresponding axes of $\mathrm{K}_{\mathrm{e}}(t)$ and $\mathrm{K}_{\mathrm{e}}(t+d t)$ remain parallel to each other at any $t$. Hence, the rotational frequency of the electron spin $\omega_{s}$, measured in $\mathrm{K}_{\mathrm{e}}$, coincides with the Larmor frequency $\omega_{L}$ of precession of the magnetic dipole moment of electron around the magnetic field of the nucleus, i.e.:

$$
\omega_{s}=\omega_{L}\left(\mathrm{~K} \rightarrow \mathrm{K}_{\mathrm{e}}(t) \rightarrow \mathrm{K}_{\mathrm{e}}(t+d t)\right) .
$$

Concurrently, we notice that the transformations $\mathrm{K} \rightarrow \mathrm{K}_{\mathrm{e}}(t) \rightarrow \mathrm{K}_{\mathrm{e}}(t+d t)$ entail the relative spatial rotation of the systems $\mathrm{K}$ and $\mathrm{K}_{\mathrm{e}}(t+d t)$ at the Thomas-Wigner angle $d \theta$ around the axis $z$. Hence, the observers in the electron's frames $\mathrm{K}_{\mathrm{e}}(t)$ and $\mathrm{K}_{\mathrm{e}}(t+d t)$ see different spatial orientations of the $x$ - and $y$ - axes of the laboratory system $\mathrm{K}$, which differ from each other by the angle $-d \theta$. This means that in $\mathrm{K}_{\mathrm{e}}$, the system $\mathrm{K}$ rotates around the axis $z$ with the frequency

$$
\omega_{K}=-\frac{d \theta}{d t}=-\omega_{T}=-\frac{\omega_{L}}{2}\left(\mathrm{~K} \rightarrow \mathrm{K}_{\mathrm{e}}(t) \rightarrow \mathrm{K}_{\mathrm{e}}(t+d t)\right)
$$

Equations (1) and (2), obtained under the choice of successive LT $\mathrm{K} \rightarrow \mathrm{K}_{\mathrm{e}}(t) \rightarrow \mathrm{K}_{\mathrm{e}}(t+d t)$, indicate that in the inertial frames, co-moving with the orbiting electron at different time moments, the precession of its spin happens with the Larmor frequency $\omega_{L}$ (eq. (1)), and no "Thomas half" is present in the frames $\mathrm{K}_{\mathrm{e}}(t)$ at any $t$, unlike the precession frequency $\omega_{L} / 2$ for a laboratory observer.

Thus we conclude that the succession of transformations $\mathrm{K} \rightarrow \mathrm{K}_{\mathrm{e}}(t) \rightarrow \mathrm{K}_{\mathrm{e}}(t+d t)$ leads to the result, which is not adequate from the relativistic viewpoint.

Next, we consider the rotation-free $\mathrm{LT} \mathrm{K} \rightarrow \mathrm{K}_{\mathrm{e}}(t)$, $\mathrm{K} \rightarrow \mathrm{K}_{\mathrm{e}}(t+d t)$ and analyze their implications in the electron's frame.

This choice of transformations implies, by definition, no relative rotation between the systems $\mathrm{K}$ and $\mathrm{K}_{\mathrm{e}}(t)$ at any $t$. Therefore, observers in co-moving electron's frames $\mathrm{K}_{\mathrm{e}}(t)$ see fixed spatial orientations of the axes of the labframe $\mathrm{K}$ at any $t$, which means that no rotation of the system $\mathrm{K}$ takes place in $\mathrm{K}_{\mathrm{e}}$, i.e.

$$
\omega_{K}=0\left(\mathrm{~K} \rightarrow \mathrm{K}_{\mathrm{e}}(t), \mathrm{K} \rightarrow \mathrm{K}_{\mathrm{e}}(t+d t)\right) .
$$

instead of eq. (2), derived under the alternative choice of succession of rotation-free LT.

Further, we point out that under the rotation-free transformations $\mathrm{K} \rightarrow \mathrm{K}_{\mathrm{e}}(t), \mathrm{K} \rightarrow \mathrm{K}_{\mathrm{e}}(t+d t)$, the $x$ - and $y$-axes of the frame $\mathrm{K}_{\mathrm{e}}(t+d t)$ are turned out with respect to the axes of $\mathrm{K}_{\mathrm{e}}(t)$ at the Thomas-Wigner angle $d \theta_{T-W}$ around the axis $z$. Hence, in $\mathrm{K}_{\mathrm{e}}(t)$, the electron spin also experiences this rotation, which should be added to its rotation due to Larmor precession. Therefore, in the frame $\mathrm{K}_{\mathrm{e}}$, the resultant rotation of the electron's spin happens with the half of the frequency $\omega_{L}$ :
$\omega_{s}=\omega_{L}-\frac{d \theta_{T-W}}{d t}=\omega_{L}-\omega_{T}=\frac{\omega_{L}}{2}\left(\mathrm{~K} \rightarrow \mathrm{K}_{\mathrm{e}}(t), \mathrm{K} \rightarrow \mathrm{K}_{\mathrm{e}}(t+d t)\right)$. (4)

Thus, we conclude that under the choice of rotation-free $\mathrm{LT} \mathrm{K} \rightarrow \mathrm{K}_{\mathrm{e}}(t), \mathrm{K} \rightarrow \mathrm{K}_{\mathrm{e}}(t+d t)$, both a laboratory observer and an observer, co-moving with the electron at any time moment $t$, see the same frequency (4) of precession of electron's spin.

The analysis implemented above allows asserting that only the succession of rotation-free $\mathrm{LT} \mathrm{K} \rightarrow \mathrm{K}_{\mathrm{e}}(t), \mathrm{K} \rightarrow \mathrm{K}_{\mathrm{e}}(t+d t)$ ensures relativistically adequate solutions for the spin-orbit interval, derived in the semi-classical approach to hydrogenlike atom, both in the labframe $\mathrm{K}$ and in the proper electron frames $\mathrm{K}_{\mathrm{e}}(t)$.

Concurrently, we have seen that the alternative choice of succession of transformations $\mathrm{K} \rightarrow \mathrm{K}_{\mathrm{e}}(t) \rightarrow \mathrm{K}_{\mathrm{e}}(t+d t)$ yields the incorrect equation (1) in the frame $\mathrm{K}_{\mathrm{e}}$ and should be rejected.

This result shows that in relativistic problems, dealing with successive LT between at least three Lorentz frames, moving with non-collinear relative velocities, the order of application of these transformations cannot be made arbitrarily at least in the case, where two of the frames under consideration represent the proper frames co-moving with the same object at different time moments. It is obvious that this statement is valid not only for a circular motion, considered in the analysis of Thomas precession, but for any motion along a smooth curved path, too, because at any spatial point, such a motion can be approximated by a momentary circular motion with a suitable radius.

From the physical viewpoint, this result signifies that, as soon as we set the rotation-free LT between the frame of observation $\mathrm{K}$ and the frame $\mathrm{K}(t)$ co-moving with a tracking object at the time moment $t$, then the rotation-free transformation between $\mathrm{K}$ and $\mathrm{K}(t)$ remains in force at future time moments, too.

In our opinion, this property of LT, which we suggest to name the "tracking rule", represents an important addition to the available mathematical structure of special theory of relativity (STR), which allows avoiding non-adequate solutions of relativistic problems, dealing with successive LT, as we have demonstrated above via the analysis of Thomas precession.

In a more general view, let us show that the "tracking rule" represents the necessary condition for covariant description of the motional equation for spin, which is given by the BMT equation [29]. Indeed, the spatial components of BMT equation for a charged particle with spin $s$, moving along a curved path, can be presented in the form (see, e.g. [22])

$$
\left(\frac{d \mathbf{s}}{d t}\right)_{\mathrm{lab}}=\left(\frac{d \mathbf{s}}{d t}\right)_{\mathrm{co-moving}}+\omega_{T} \times \boldsymbol{s},
$$

where the subscript "lab" defines the quantities in a laboratory frame, the subscript "co-moving" defines the quantities in a set of the frames co-moving with the charged particle, while 
the last term on rhs describes the rotation of the systems attached to the particle, which, we emphasize, is only possible under application of the "tracking rule", which ensures the Thomas-Wigner rotation between the systems $\mathrm{K}(t)$ and $\mathrm{K}(t+d t)$, attached to the particle at the time moments $t$ and $t+d t$, correspondingly.

Thus, we conclude that this rule represents the necessary element for covariant description of motion of charged particles with spin, and thus, the choice of successive rotationfree $\mathrm{LT} \mathrm{K} \rightarrow \mathrm{K}_{\mathrm{e}}(t) \rightarrow \mathrm{K}_{\mathrm{e}}(t+d t)$ in the description of hydrogenlike atom [1, 2, 5, 21, 23-26], being at odds with the "tracking rule", actually leads to relativistically non-adequate result (1) in the frames $\mathrm{K}(t)$.

Further on, we highlight the general character of eq. (5), which is applicable not only to electron's spin, but also remains in force for any vector, describing, e.g., the motion of macroscopic gyroscope [30]. In a macroscopic scale, it seems especially important to carry out the direct measurement of the Thomas-Wigner rotation with respect to macroscopic extended bodies moving in a laboratory frame, which will have the fundamental significance as the new experimental verification of relativity theory in phenomena, dealing with successive space-time transformations.

In addition, we show in the next section, that such measurements will open the possibility to develop new astrometric methods, which will allow, via the tracking rule, to extract unique information with respect to the most general modes of motion of Earth and the Sun in the Universe.

\section{Thomas-Wigner rotation of elongated} macroscopic objects in terrestrial conditions and its possible application to astrometric measurements. In this section we deal with macroscopic objects, moving along curved paths, and focus our attention to the effect of Thomas-Wigner rotation under application of the "tracking rule", assuming that the variation of the angle $\theta_{T-W}$ with time (i.e., the Thomas precession) is very slow; this is actually the case, e.g., for celestial bodies moving under their gravitational interaction.

For the simplest one-body problem, where an object with the rest mass $m$ orbits a host body with practically infinite mass, the motion of the object $m$ along an elliptic orbit immediately provides a natural choice of rotation-free LT between the frame $\mathrm{K}$, attached to the host body, and the frame $\mathrm{K}(t)$, co-moving with an orbiting object at the considered moment $t$. According to the tracking rule, this choice of rotation-free transformations remains in force in that case, where an object, moving along an elliptic orbit, simultaneously experiences additional movement (e.g. small oscillations near its non-disturbed positions). As an example, in Fig. 2 we show the Lorentz frame $\mathrm{K}_{1}$, co-moving at the considered time moment with an object 1 , orbiting in the $x y$ plane along the elliptic orbit around the immovable host body, as well as the Lorentz frame $\mathrm{K}_{2}$, co-moving with another object 2, which, in addition to its elliptic motion around the same host body, continuously oscillates in the same plane with a small amplitude near its current equilibrium position. We assume that the distance between the objects 1 and 2 is much smaller than their distances from the host body, so that their orbital velocity $\boldsymbol{v}$ is practically the same.

Following the "tracking rule", we designate the rotationfree $\mathrm{LT}$ from $\mathrm{K}$ to $\mathrm{K}_{1}$ via $\mathbf{L}(\boldsymbol{v})$, and the rotation-free $\mathrm{LT}$ from $\mathrm{K}$ to $\mathrm{K}_{2}$ via $\mathbf{L}(\boldsymbol{v} \oplus \boldsymbol{u})$, where $\boldsymbol{u}$ is the velocity of oscillation motion of the object 2. Hence, the successive LT from $K_{1}$ to $K_{2}$ acquire the form $\mathbf{L}^{-1}(\boldsymbol{v}) \mathbf{L}(\boldsymbol{v} \oplus \boldsymbol{u})$. In comparison with the direct rotation-free transformation $\mathbf{L}(\boldsymbol{u})$ between $\mathrm{K}_{1}$ and $\mathrm{K}_{2}$, the transformation $\mathbf{L}^{-1}(\boldsymbol{v}) \mathbf{L}(\boldsymbol{v} \oplus \boldsymbol{u})$ entails the Thomas-Wigner rotation between these frames in the $z$-direction. To the accuracy of calculations $c^{-2}$, the angle of this rotation is equal to (e.g., [22])

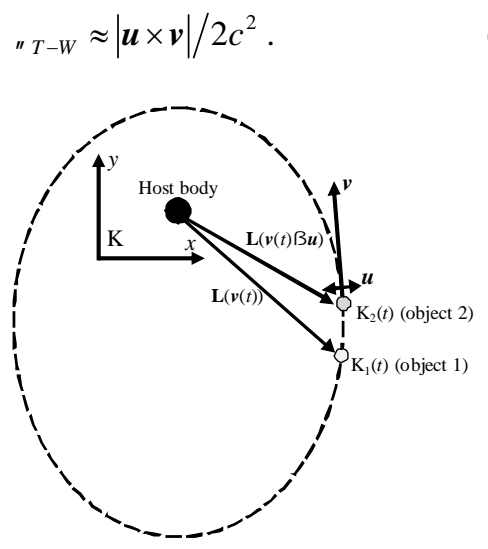

Fig. 2: The object 1 with its Lorentz frame $K_{1}$ and the object 2 with its Lorentz frame $K_{2}$ are moving along the same elliptic orbit around the immovable host body (the frame $\mathrm{K}$ ) in the $x y$-plane. The object 2, in addition to its elliptic motion, continuously oscillates in the rotational plane near its current elliptic coordinate at the velocity $\boldsymbol{u}$, which is orthogonal to its orbital velocity $\boldsymbol{v}$. We assume that $u<<v$, and the distance between the objects 1 and 2 is much smaller than their respective distances from the host body and their orbital velocity $\boldsymbol{v}$ is practically the same. According to the "tracking rule", we carry out the rotation-free $\mathrm{LT} \mathbf{L}(\boldsymbol{v})$ from $\mathrm{K}$ to $\mathrm{K}_{1}$ and the rotation-free LT $\mathbf{L}(\boldsymbol{v} \oplus \boldsymbol{u})$ from $\mathrm{K}$ to $\mathrm{K}_{2}$, so that the LT from $\mathrm{K}_{1}$ to $\mathrm{K}_{2}$ is not rotation-free.

We emphasize that the angle (6), at least in principle, can be measured by an observer in the frame $K_{1}$ (which can be considered as the labframe on the surface of Earth). Hence, having known the angle $\theta_{T-W}$, and assuming the velocity $\boldsymbol{u}$ to be known at any time moment, we further evaluate according to (6) the component of velocity $\boldsymbol{v}_{\perp}$ of Earth orthogonal to $\boldsymbol{u}$.

The importance of such measurement becomes clearly seen in the situation, where an observer in the labframe $\mathrm{K}_{1}$ knows nothing about the host body, which involves his/her frame into the elliptic motion.

More specifically, a terrestrial observer can use the fact of rotation of Earth around the Sun and decide that the rotationfree LT between the Sun and Earth $\left(\mathbf{L}_{\mathrm{S}-\mathrm{E}}\right)$ is applicable at any 
time moment. At the same time, the Sun is also involved into a rotational motion in our Galaxy, so that the actual rotation-free LT is realized between the frames attached to the Galaxy rotational center and the Sun $\left(\mathbf{L}_{\mathrm{G}-\mathrm{S}}\right)$. However, in such a case, the successive transformations from the Galaxy rotational center to Earth $\left(\mathbf{L}_{\mathrm{G}-\mathrm{S}} \mathbf{L}_{\mathrm{S}-\mathrm{E}}\right)$ would not be rotation-free, which does contradict the tracking rule, according to which the LT from the Galaxy rotational center to Earth must be rotationfree. Thus, in order to avoid this apparent contradiction, one has to adopt that the LT from the frame of the Sun to the frame of Earth cannot be rotation-free, as soon as the Sun is involved into its Galactic motion. In this situation, the measured Thomas-Wigner angle (6) should correspond to the maximal value of $\boldsymbol{v}_{\perp}$, which represents the velocity of Earth in the Galaxy.

A sketch of the simplest experimental scheme for measurement of the angle (6) is shown in Fig. 3, where some rod of the proper length $l$, oriented along the axis $x$, moves along the axis $y$, and at the time moment, when the rod intersects the axis $x$ of the laboratory frame, its velocity for a laboratory observer is equal to $u$.

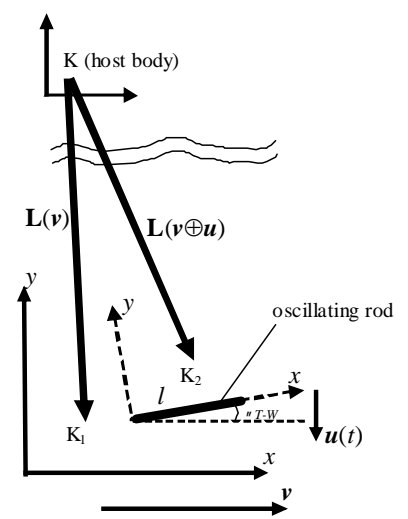

Fig. 3: Further details of Figure 2: the laboratory frame $K_{1}$ is located on Earth, and the rod $l$ oriented along the $x$-axis experiences reciprocating motion along the axis $y$ with the velocity $u$ at the moment of its intersection with the axis $x$. Due to the Thomas-Wigner rotation of the rest frame of the $\operatorname{rod} K_{2}$ with respect to $K_{1}$, the opposite ends of the rod intersect the axis $x$ at different time moments of the frame $\mathrm{K}_{1}$, which is equipped with corresponding apparatus aimed to measure this time difference.

Linking Figs. 2 and 3, we designate the laboratory frame as $\mathrm{K}_{1}$, and the co-moving frame of the rod at the moment of its intersection with the $x$-axis as $\mathrm{K}_{2}$. Thus, due to the "tracking rule", the systems $K_{1}$ and $K_{2}$ experience the Thomas-Wigner rotation around the axis $z$ at the angle (6). Therefore, the intersection of the rod with the axis $x$ of the frame $\mathrm{K}_{1}$ happens at different time moments for its different points. One should notice that in typical laboratory conditions, where, e.g., the length of the rod $l$ is near $1 \mathrm{~m}$, and a typical velocity of the Sun in the Universe $v \approx 10^{-3} c$ (where $c$ is the light velocity in vacuum), the corresponding time difference is tiny. Indeed, at the Thomas-Wigner angle $\theta_{T-W}=u v / 2 c^{2}$, it is equal to

$$
\Delta t=\frac{l \theta_{T-W}}{u}=\frac{l v}{2 c^{2}} \approx 10^{-12} \mathrm{~s} .
$$

In these conditions, applying reciprocating motion of the rod, we get the possibility of repeating measurements of the time difference (6). Then, using modern optical methods in combination with the appropriate data processing procedures, it is possible to measure the time interval (7) even under its fluctuations near the average value caused by vibrations in the rod and other factors, distorting its motion. In these conditions, a self-rotation of Earth, leading to daily variation of the angle between vectors $\boldsymbol{v}$ and $\boldsymbol{u}$ and corresponding variation of the time difference (7) can be considered as a factor, which will simplify the interpretation of the results obtained.

At the same time, a detailed discussion with respect to real technical approaches to the performance of this experiment lies outside the scope of the present paper.

One should notice that the measurement of the ThomasWigner rotation in the experiments of macroscopic scale had never been realized before, because intuitively it was natural to adopt that the rest frame of any elongated object, moving with respect to a laboratory observer, should be related to the laboratory frame via the rotation-free LT, where no ThomasWigner rotation of this object in the laboratory frame is expected. Thus, only the disclosure of the "tracking rule", which invalidates this supposition and implies a more complicated transformation from the laboratory frame to the proper frame of a moving object, makes topical the proposed experiment.

As an outcome of such measurements, we conjecture to be able to get the possibility to evaluate the velocity of Earth and the Sun is space, associated with the component of their elliptic motion in the Universe, and to compare it, e.g., with the known velocity of the Sun in the frame of isotropy of cosmic relic radiation.

4. Conclusion. - The presented approach to the Thomas precession and determination of spin-orbit interval in the hydrogenlike atom allowed us to conclude that the two possible successions of rotation-free $\mathrm{LT}, \mathrm{K} \rightarrow \mathrm{K}_{\mathrm{e}}(t) \rightarrow \mathrm{K}_{\mathrm{e}}(t+d t)$ and $\mathrm{K} \rightarrow \mathrm{K}_{\mathrm{e}}(t), \mathrm{K} \rightarrow \mathrm{K}_{\mathrm{e}}(t+d t)$, are, in general, not equivalent to each other. We have shown that only the set of transformations $\mathrm{K} \rightarrow \mathrm{K}_{\mathrm{e}}(t), \quad \mathrm{K} \rightarrow \mathrm{K}_{\mathrm{e}}(t+d t)$ provides relativistically adequate solutions both in the laboratory frame and in the rest frames of the orbiting electron and, by such a way, we came to the "tracking rule", where the rotation-free LT between the frame of observation $\mathrm{K}$ and the frame $\mathrm{K}(t)$ co-moving with a tracking object at the initial time moment, remains in force at any future time moments, too.

The disclosed "tracking rule" represents an important addition to the physical content and mathematical structure of STR, and is closely related to the implementation of eq. (5), resulting from the covariant BMT equation for spin. From this 
angle of view, the "tracking rule" directly stems from the covariance principle, which ensures relativistically adequate solutions in any frames of observation realizable in Nature.

From the practical viewpoint, the "tracking rule" allows to eliminate in many situations any ambiguities in the choice of physically meaningful successions of rotation-free transformations between three Lorentz frames under consideration and, by such a way, straightforwardly resolves the known paradoxes with respect to the Thomas precession and Thomas-Wigner rotation (e.g., [5, 17, 19, 31]. The explicit resolution of these paradoxes will be done elsewhere.

An attractive perspective of application of the tracking rule is opened with respect to the determination of the most fundamental modes of motion of the Sun in the Universe, via the measurement of the Thomas-Wigner rotation of an elongated object (e.g., the rod in Fig. 3), moving in the laboratory frame.

We emphasize that the possibility of such measurements even if the estimated velocity of the Sun will agree with its velocity in the frame of isotropy of cosmic relic radiation does not create any doubts in the validity of the Einstein relativity principle. Indeed, the "tracking rule", laying on the basis of such measurements, is established only with respect to motional diagrams, where some of the objects under consideration move along curved paths, so that the possibility to reveal the motion of one frame with respect to another frame via "internal" measurement procedure (e.g., the Thomas-Wigner rotation of the moving body) does not touch the validity of Einstein relativity principle formulated in effect for strictly inertial motion.

In the case, where the relative velocities between three inertial frames $\boldsymbol{v}, \boldsymbol{u}$ and $\boldsymbol{v} \oplus \boldsymbol{u}$ do not depend on time, the "tracking rule" is no longer needed, and the succession of rotation-free LT between these frames is set exogenously. In such a case, the presence of time-independent Thomas-Wigner rotation between two inertial systems does not bring nontrivial information and straightforwardly follows from the general group properties of LT.

\section{REFERENCES}

[1] ThOMAS L.H., Nature 117 (1926) 514

[2] ThOMAS L.H., Phil. Mag. 7 (1927) 1.

[3] Wigner E.P., Ann. Math. 40 (1939) 149.

[4] UhLENBECK G.E., GoudsMIT S., Nature 117 (1926) 264.

[5] KenNedy W.L., Eur. J. Phys. 23 (2002) 235.

[6] KröMer H., Am. J. Phys. 78 (2004) 51.

[7] Malykin G.B., Physics-Uspekhi 49 (2006) 837.

[8] Lush D.C., Am. J. Phys. 78 (2010) 1422.

[9] O'Donnell K., Visser M., Eur. J. Phys. 32 (2011) 1033.

[10] Dragan A., Odrzygozdz T., Am. J. Phys. 81 (2013) 631.

[11] Spavieri G., Mansuripur M., Phys. Scr. 92 (2015) 085501.

[12] Silenko A.J., Phys. Rev. D93 (2016) 124050.
[13] Spavieri G., Eur. Phys. J. D70 (2016) 263.

[14] KHOLMETSKII A.L., MISSEVITCH O.V., Yarman T., Am. J. Phys. 78 (2010) 428.

[15] KHOLMETSKII A.L., MISSEVITCH O.V., YARMAn T., Am. J. Phys. 78 (2010) 1423.

[16] Kholmetskil A.L., Yarman T., Can. J. Phys. 92 (2014) 1232.

[17] KHOLMETSKII A.L., MisSEVITCH O.V., YARMAn T., Can. J. Phys. 92 (2014) 1380.

[18] Kholmetskil A.L., Yarman T., Can. J. Phys. 93 (2015) 503.

[19] KhOlMETSKII A.L., YARMAN T., Eur. Phys. J. Plus 132 (2017) 400.

[20] KHOLMETSKII A.L., MisSEVITCH O.V., YARMAn T., Ann. Phys. 384 (2017) 155.

[21] MøLlER C., The Theory of Relativity (Clarendon Press, Oxford) 1973.

[22] JACKSON J.D., Classical Electrodynamics, 3rd ed. (Wiley, New York) 1998.

[23] SOMMERfEld A., Atombau und Spektrallinien (Braunschweig, Vieweg) 1931.

[24] Belloni L., Reina C., Eur. J. Phys. 7 (1986), 55.

[25] Yamasaki H., Eur. J. Phys. 12 (1991) 218.

[26] Munoz G., Am. J. Phys. 69 (2000) 554.

[27] Goedicke G.H., Am. J. Phys. 46 (1978) 1055.

[28] Farach H.A., Aharonov Y., Ch.P. PoOle, Jr., Am. J. Phys. 47 (1979) 247.

[29] BARgMANN V., Michel L., TElegdi V.L., Phys. Rev. Lett. 2 (1959) 435.

[30] GoldsteIn H., Classical Mechanics 2nd ed. (Addison-Wesley) 1980.

[31] BACRY H., Nuovo Cimento 26 (1962) 1164. 